\title{
LASER SPECTROSCOPY OF NEUTRAL ATOMS IN SUPERFLUID HELIUM: NEW ENVIRONMENT FOR SPECTROSCOPY AND CHEMISTRY
}

\author{
QIN HUI, JOHN L. PERSSON, JAAP H. M. BEIJERSBERGEN \\ and MICHIO TAKAMI \\ The Institute of Physical and Chemical Research (RIKEN) \\ Wako, Saitama 351-01, Japan
}

(Received 18 April, 1994)

\begin{abstract}
The optical spectra of neutral atoms are studied in superfluid helium (HeII). Neutral atoms are dispersed into HeII by laser ablation of solid samples immersed in HeII. Absorption and emission spectra of dispersed atoms are studied with pulsed dye lasers. Ytterbium is found to be a typical bubble atom which resides in a bubble-like cavity formed by a strong repulsive force against the surrounding $\mathrm{He}$ atoms. Resonance quenching of LIF by accidental overlap of the resonant induced emission and broad absorption lines is observed.
\end{abstract}

KEY WORDS: superfluid helium, bubble atom, LIF, resonance quenching

\section{INTRODUCTION}

Liquid helium has several unusual properties compared to other solvent liquids. It is stable below $4.2 \mathrm{~K}$ under $1 \mathrm{~atm}$, and becomes superfluid below $2.2 \mathrm{~K}$ (HeII) where the fluid has a very small viscosity and extremely high thermal conductivity. However, important characteristics of liquid helium as a solvent originate from a small polarizability of He atom. Firstly, a strong repulsive force due to Pauli exclusion principle and a very weak attractive force between the He and dispersed atoms frequently result in the formation of a bubble-like cavity around the atom.' Secondly, liquid helium hardly dissolves atoms and molecules spontaneously because the small polarizability does not stablize the dispersed particles, and also because the temperature is very low. As a consequence, spectroscopy and chemistry of atoms and molecules in liquid helium is still scarce compared to the work in other rare gas liquids ( $\mathrm{Ar}, \mathrm{Kr}$ and $\mathrm{Xe}$ ), in which substantial amount of studies on the infrared spectroscopy and photochemistry have been reported. ${ }^{2}$

Laser spectroscopy of impurity atoms in HeII was first reported in 1986 for $^{\mathrm{Ba}^{+}}$ implanted into the liquid, ${ }^{3}$ and subsequently for the neutral atoms produced by recombination of implanted atomic ions with electrons dispersed simultaneously into the 
liquid., ${ }^{4,5}$ Quite recently, Yabuzaki and his co-workers reported a new and more versatile experimental technique, laser ablation of solid samples immersed in HeII to disperse atoms and molecules in the liquid. ${ }^{6}$ The present letter reports our study of the properties of neutral atoms, in particular of $\mathrm{Sr}$ and $\mathrm{Yb}$, in HeII produced by the laser ablation method.

\section{EXPERIMENTAL METHOD}

Figure 1 shows our experimental arrangement. ${ }^{7}$ Superfluid helium was produced in a Pyrex double bath dewar by reducing the vapor pressure to about 10 torr above the liquid. Solid samples were fixed on an aluminum plate and placed near the bottom of the dewar. A pulsed YAG laser beam $(10-20 \mathrm{~Hz}, 2$ nd or $3 \mathrm{rd}$ harmonics, $10-20 \mathrm{~mJ} / \mathrm{pulse}$ ) was focused onto the sample with a quartz lens $(\mathrm{f}=1 \mathrm{~m})$. The laser ablation produces a large number of small particles of the sample material in the liquid. These particles were dissociated with a second pulsed YAG laser beam to disperse neutral atoms and molecules into the liquid. ${ }^{6}$ Pulsed dye lasers, pumped by an excimer or YAG laser, were used to probe the dispersed atoms. The pulsed dye laser beam, which was unfocused to avoid non-linear process such as multiphoton absorption, was merged with the dissociation laser beam spatially. The delay times among the three laser pulses are variable over a wide range. Typical values are a few $\mu \mathrm{s}$ for the ablation and dissociation lasers, and $50 \mathrm{~ns}$ to $10 \mathrm{~ms}$ between the dissociation and dye lasers. All the laser beams were introduced through quartz win-

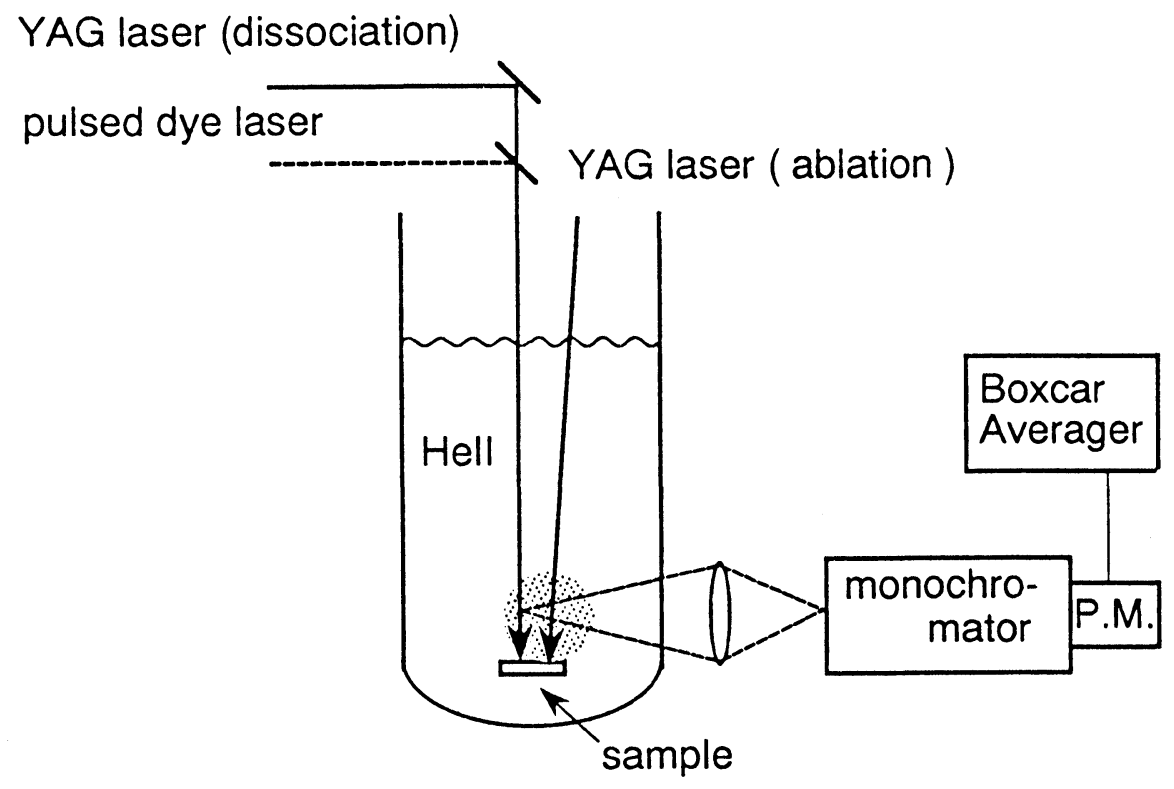

Figure 1 Experimental arrangement. 
dows on the top flange of the dewar. The emission from the atoms was focused onto a slit of $25 \mathrm{~cm}$ monochromator and detected with a photomultiplier. The pulsed signal was averaged with a Boxcar integrator. The wavelengths of the dye lasers were calibrated against optogalvanic signals of $\mathrm{Ne}$ in a hollow cathode lamp. The monochromator, which has $0.5 \mathrm{~nm}$ resolution, was calibrated with the dye lasers.

The optical spectra of impurity atoms were measured by several different experimental arrangements. The small particles produced by laser ablation remain in the liquid for a considerable length of time (1/2-1 hour). After ablating the sample for 10-20 min., the liquid contains enough amount of small particles, allowing the observation of LIF signal only with the dissociation and probe lasers. This long life time of the particles also allows us to measure LIF only with the ablation and probe lasers, where the ablation laser functions as a dissociation laser as well. The dissociation of small particles is associated with an optical emission, which we will call direct emission hereafter. The direct emission is believed to be the recombination emission of ions and electrons in a plasma-like state produced by the dissociation laser.

\section{EXPERIMENTAL RESULTS AND DISCUSSION}

\section{Direct emission}

An example of the direct emission spectrum of $\mathrm{Sr}$ is shown in Fig. 2. The spectrum was taken by scanning the monochromator while ablating the Sr sample with the 3rd harmonics of YAG laser. Strontium has two valence electrons, forming singlet and triplet manifolds. The observed spectrum contains very strong lowest singlet $5 s 5 p{ }^{1} \mathrm{P}_{1}^{o} \rightarrow 5 s^{2}{ }^{1} \mathrm{~S}_{\mathrm{o}}(460.9 \mathrm{~nm})$ and lowest triplet $5 s 6 s{ }^{3} \mathrm{~S}_{1} \rightarrow 5 s 5 p{ }^{3} \mathrm{P}_{0-2}^{o}(679-707$ $\mathrm{nm})$ transitions, and also some higher triplet transitions. However, close examination of weaker lines reveals some new features of the direct emission. One such example is the weak emission lines observed around $408 \mathrm{~nm}$. The relative intensity and spacing of two lines coincide almost exactly with the lowest $5 p \rightarrow 5 s$ transitions of $\mathrm{Sr}^{+}$, clearly indicating the production of excited ions during the dissociation process. Another example is the weak lines observed around $550 \mathrm{~nm}$. The relative intensity and line positions are in good agreement with the $4 d 5 p^{3} \mathrm{D}_{1-3}^{\mathrm{o}} \rightarrow 5 s 4 d^{3} \mathrm{D}_{1-3}$ transitions in free space. For the lowest singlet transition, the direct emission line is confirmed to be the emission from a bubble state. ${ }^{7}$ However, some atoms, in particular alkali metal atoms, are known to emit radiation from a non-bubble state. It is not confirmed yet whether these weaker lines originate from bubble states or not.

\section{Bubble bands}

The stability of bubble state is based on the surface energy of liquid helium, which is given by $4 \pi \sigma R^{2}$ where $\sigma=2.2 \times 10^{11} \mathrm{eV} / \mathrm{cm}^{2}\left(1.8 \times 10^{15} \mathrm{~cm}^{-1} / \mathrm{cm}^{2}\right),{ }^{8}$ and $R$ is a bubble radius. When the attractive force is weak, the combination of a repulsive force and the surface energy gives rise to the potential which has a shallow potential 


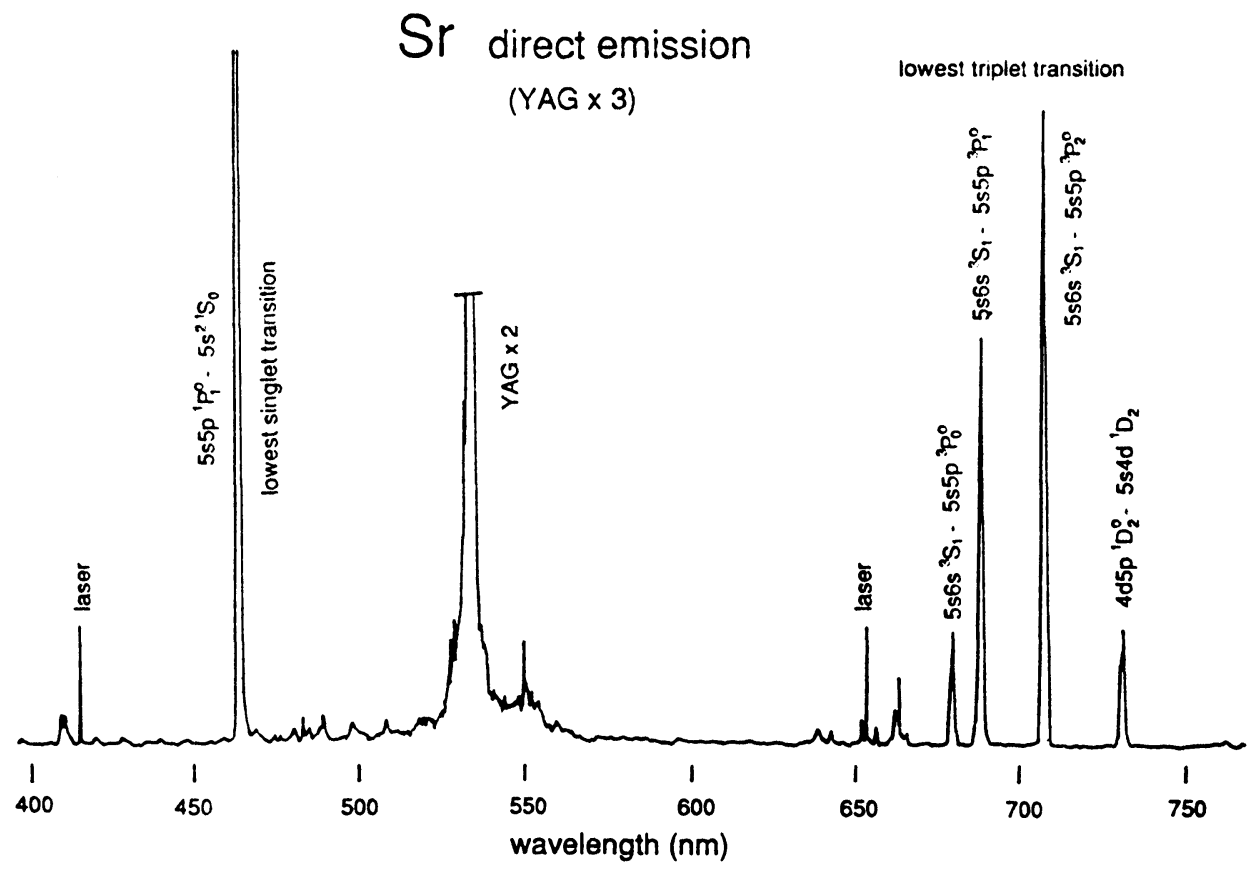

Figure 2 Direct emission spectrum of Sr. The third harmonics of YAG laser was used for ablation and dissociation. The spectrum contains several sharp laser emission lines.

minimum as shown in Fig. 3. In general, this minimum is a function of the angular variables of spherical coordinates in three dimensional space and forms a closed non-spherical surface. Then angular-dependent radial vibrational motions exist around the equilibrium position of the surface. We call this motion as bubble vibrations or bubble modes. When a valence electron of the bubble atom is excited, the increase in mean radius of the electron orbit increases the bubble radius.

The bubble state has been observed for alkaline earth atoms, ${ }^{5,7} \mathrm{Ca}, \mathrm{Sr}, \mathrm{Ba}$, and heavy alkali metal atoms, ${ }^{9} \mathrm{Rb}$ and $\mathrm{Cs}$. In our previous study, ${ }^{7}$ no bubble state was identified in $\mathrm{Yb}$, which has an electronic configuration similar to alkaline earth atoms. Recently, however, we found that $\mathrm{Yb}$ is also a typical bubble atom. Figure 4 shows one of the lowest triplet absorption (excitation) bands of $\mathrm{Yb}, 6 s 7 s{ }^{3} \mathrm{~S}_{1} \leftarrow 6 s 6 p{ }^{3} \mathrm{P}_{2}^{0}$ at $770 \mathrm{~nm}$ in free space by monitoring the emission in the $6 s 7 s{ }^{3} \mathrm{~S}_{1} \rightarrow 6 s 6 p{ }^{3} \mathrm{P}_{0}^{0}$ transition at $649 \mathrm{~nm}$. The relevant energy levels of $\mathrm{Yb}$ is shown in Fig. 5. The observed absorption band is very broad and largely blue-shifted, while the emission line is relatively narrow $(1-2 \mathrm{~nm})$ and is close to the free atomic line. Because the bubble absorption band is very broad, the observed absorption profile in Fig. 4 mostly reflects the wavelength dependence of the dye laser power.

This anomalous feature of the absorption band has been interpreted, at least semiquantitatively, to be due to the coupling of electronic transitions with bubble vibrational modes. The above triplet transition is from $6 p$ to $7 s$, where the principal quantum number, $n$, increases by one. The electronic transition occurs from the bot- 


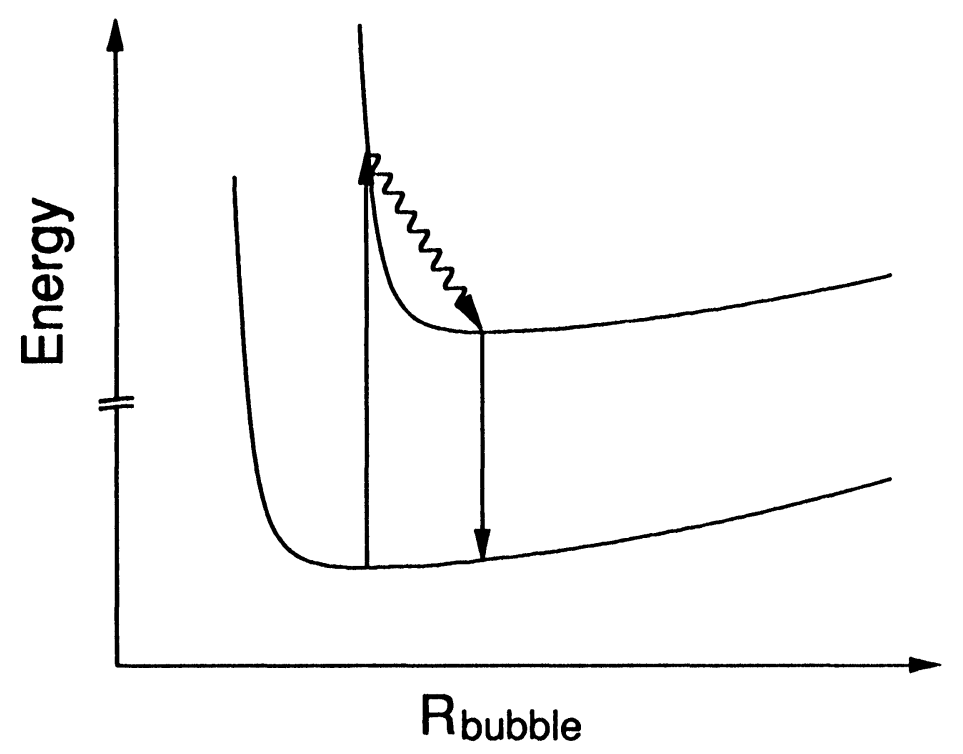

Figure 3 Potential curves for the breathing bubble mode in the ground and excited electronic states of atoms. The upper potential is shifted to the right due to the increase in the mean radius of valence electron by excitation. The optical absorption occurs vertically to the sharp rising edge of the repulsive potential, producing highly compressed bubble in the excited state.

tom of the lower potential as shown in Fig. 3, because the vibrational motions are hardly excited at $1-2 \mathrm{~K}$. Then, the upper state accessible from the lower state is the sharp-rising edge of the repulsive potential because the upper state should have a larger bubble radius. Thus the absorption is largely blue-shifted. The transition intensity is determined by a Franck-Condon overlap integral of the lower and upper vibrational wave functions. This integral should have appreciable values over a wide range of the upper state energy, and produces extremely broad absorption bands. For the transition with $\Delta n=0$, the shift and width of the absorption band is much smaller as observed in the lowest singlet transitions of $\mathrm{Ca}, \mathrm{Sr}$, and $\mathrm{Ba}^{5,7}$

The laser excitation prepares an excited atom in a highly compressed bubble, or in other words, highly excited states of bubble vibrational modes. The optical emission occurs after this excited bubble modes release its energy into liquid and relax to the upper potential minimum. Because the emission occurs in the flat region of the potential, the emission lines are all narrower and closer to the free atomic lines.

\section{RESONANCE QUENCHING}

During the above measurement, we found a narrow dip around $680 \mathrm{~nm}$ in the broad absorption band as shown in Fig. 4. We interpreted this dip as a resonant quenching of the upper $6 s 7 s{ }^{3} \mathrm{~S}_{1}$ state to the lower $6 s 6 p{ }^{3} \mathrm{P}_{1}^{0}$ state by laser induced emission. This accidental coincidence occurred because the blue-shifted absorption band as- 


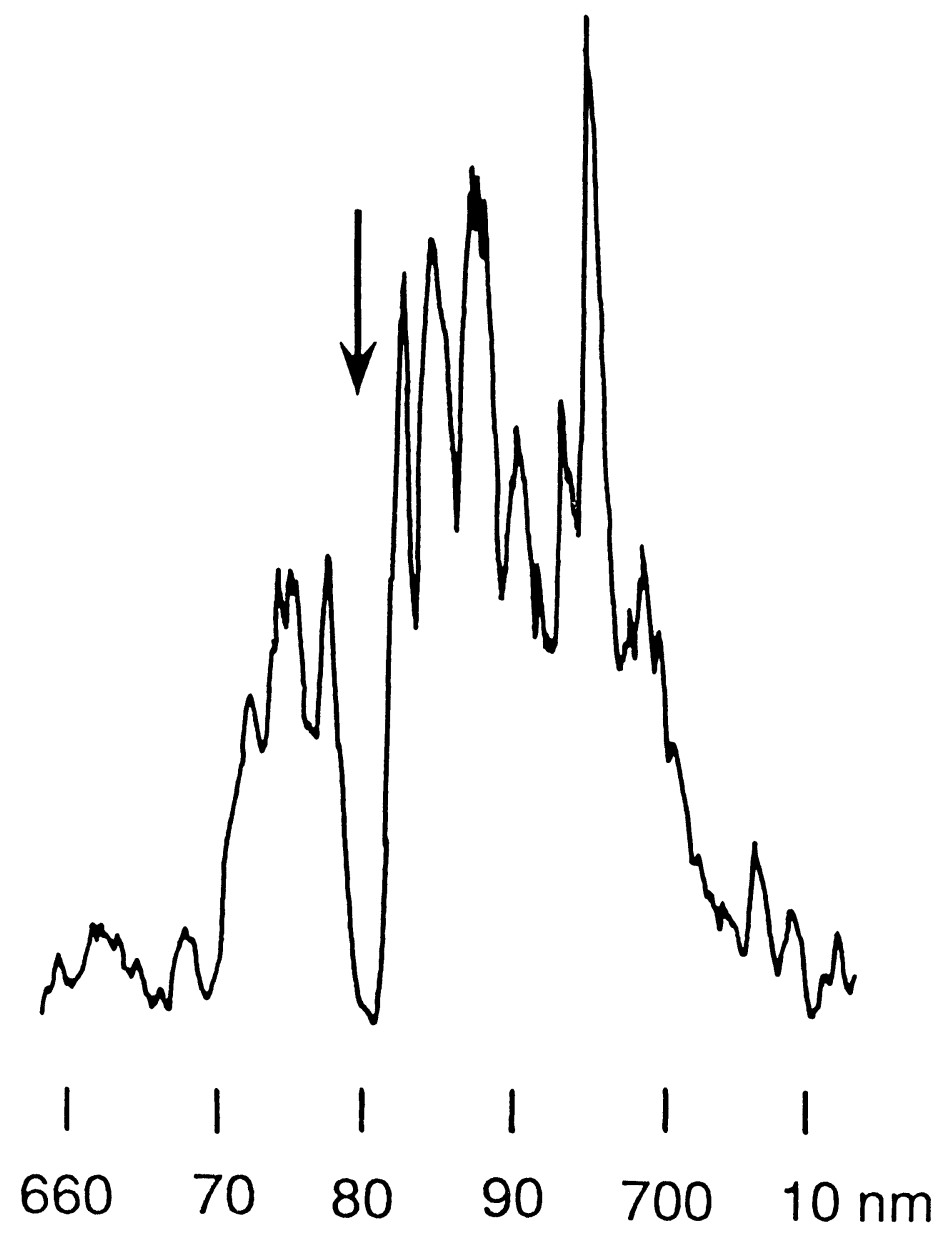

Figure 4 A bubble absorption band observed in one of the triplet transitions of $\mathrm{Yb}$. The sharp peaks in the spectrum are the noise due to the convection of the liquid in which small particles are distributed inhomogeneously. The profile does not reflect real absorption band because the signal is not normalized by the probe laser power and because the width of the band is larger than the coverage of the laser dye used. The arrow indicates the narrow dip produced by the laser-induced resonance quenching of emission.

sociated with the $6 s 7 s{ }^{3} \mathrm{~S}_{1} \leftarrow 6 s 6 p{ }^{3} \mathrm{P}_{2}^{0}$ transition happened to be accidentally overlapping with the $6 s 7 s{ }^{3} \mathrm{~S}_{1} \rightarrow 6 s 6 p{ }^{3} \mathrm{P}_{1}^{0}$ transition, inducing narrow resonant emission in this transition. The relevant energy levels are shown in Fig. 5.

This observation provides us with some important characteristics of the relaxation process of bubble modes. It should be noted that a single laser pulse excites and quenches the two transitions simultaneously, and that the quenching is almost $100 \%$. The narrow width of the hole indicates that the quenching is a resonant process, an induced emission from the bottom of the upper potential after rapid relaxation of the excited bubble modes. The complete quenching of LIF is consistent with the assumption that the bubble relaxation time is shorter than the laser pulse. Further- 


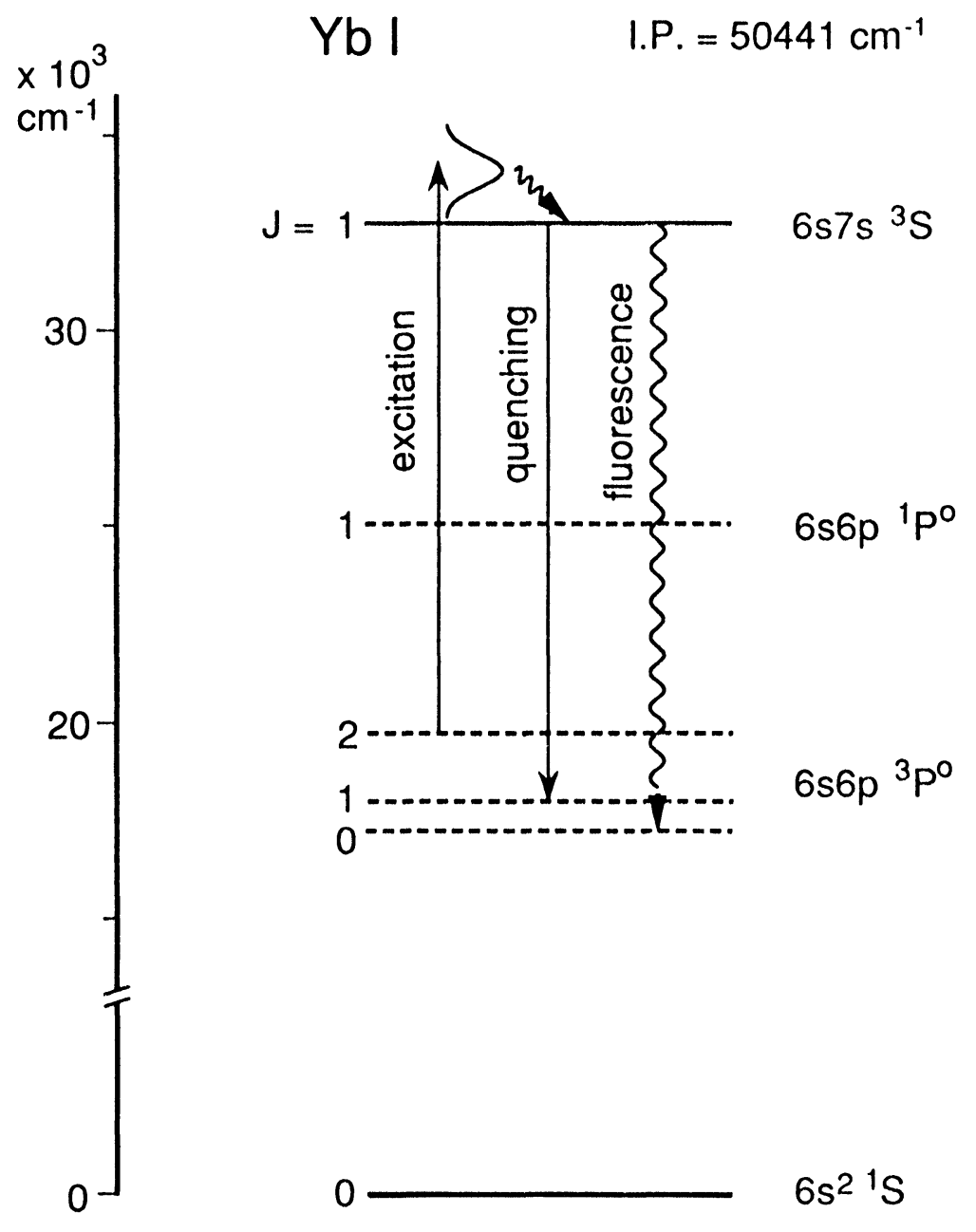

Figure 5 An energy level diagram of $\mathrm{Yb}$ relevant to the spectrum in Fig.6.

more, the lower states of induced emission also should have short relaxation time because, unless otherwise the saturation between the lower and upper states will leave finite population in the upper state, allowing fluorescence after the laser pulse.

This rapid relaxation in the lower state is consistent with the bubble model. As discussed previously, potential minimum of the upper state appears at the bubble radius larger than the value at the lower potential minimum. As a consequence, the induced emission from the bottom of upper state occurs not to the bottom of the lower state but to the states where the bubble modes are still excited to some extent as shown in Fig. 3. Thus further relaxation to the bottom of the lower potential allows total quenching of the emission. Rapid relaxation from the $6 s 6 p{ }^{3} \mathrm{P}_{1}^{\circ}$ to the $6 s 6 p{ }^{3} \mathrm{P}_{0}^{0}$ fine structure level is unlikely because the triplet-singlet emission, $6 s 6 p{ }^{3} \mathrm{P}_{1}{ }^{\circ}$ $\rightarrow 6 s^{2}{ }^{1} \mathrm{~S}_{0}$, has been observed with a lifetime in a $\mu \mathrm{s}$ range. ${ }^{10}$ 


\section{Acknowledgment}

Two authors (JLP and JHMB) are grateful for granting Science and Technology Agency (STA) Fellowship for working at RIKEN.

\section{References}

1. M. W. Cole and R. A. Bachman, Phys. Rev. B 15, 1388 (1977).

2. See, for example, J. J. Turner, M. Poliakoff, S. M. Howdle, S. A. Jackson, and J. G. Loughlin, Faraday Discuss. Chem. Soc. 86, 276 (1988).

3. H. J. Reyher, H. Bauer, C. Huber, R. Mayer, A. Schäfer, and A. Winnacker, Phys. Lett. A 115, 238 (1986).

4. H. Bauer, M. Beau, A Bernhardt, B. Friedl, and H. J. Reyher, Phys. Lett. A 137, 217 (1989).

5. H. Bauer, M. Beau, B. Friedl, C. Marchand, K. Miltner, and H. J. Reyher, Phys. Lett. A 146, 134 (1990).

6. A. Fujisaki, K. Sano, T. Kinoshita, T. Takahashi, and T. Yabuzaki, Phys. Rev. Lett. 71, 1039 (1993).

7. J. H. M. Beijersbergen, Qin Hui, and M. Takami, Phys. Lett. A 181, 393 (1993).

8. W. B. Fowler and D. L. Dexter, Phys. Rev. 176, 337 (1968).

9. Y. Takahashi, K. Sano, T. Kinoshita, and T. Yabuzaki, Phys. Rev. Lett. 71, 1035 (1993).

10. Q. Hui. J. L. Persson, J. H. M. Beijersbergen, and M. Takami, Z. Phys. B, to be published. 